\title{
Acoustic and Speech Perceptual Evaluation of health related Quality of Life among adults with Chronic Suppurative Otitis Media
}

Tahira Nomani, ${ }^{1}$ Atia Ur Rehman, ${ }^{2}$ Munaza Hayat, ${ }^{3}$ Farhan Akbar, ${ }^{4}$ Muhammad Adnan Anwar, ${ }^{5}$ Salma Ambreen Shahab, ${ }^{6}$ Muhammad Saad Faisal ${ }^{7}$

\begin{abstract}
Background: Chronic Suppurative Otitis Media, patients suffer from hearing loss causing communication problems and social withdrawal.

Objective: To assess the acoustic and speech perceptual evaluation of health related quality of life among adults with Chronic Suppurative Otitis Media.

Methodology: This cross sectional study was performed in ENT/Audiology Departments of District Headquarter Hospital, Hafizabad and Saqib Bashir General Hospital, Hafizabad, from March, 2015 to September, 2018, after the approval of synopsis. Convenient sampling method was used to induct 101 patients with Chronic Suppurative Otitis Media meeting inclusion criteria. Pure tone audiometry and speech audiometry were performed. A statement based questionnaire COMOT-15 was used to measure the scoring difference in cases.

Results: The mean age of patients was 33+10 years and $53(52.47 \%)$ were male. Out of 101 patients, the speech deficit was moderate in $7(6.93 \%)$ patients, moderately severe in $19(18.81 \%)$, severe in $42(41.58 \%)$ and speech deficit was profound in $33(32.67 \%)$ patients. There were $10(9.90 \%)$ patients with conductive type of hearing loss, $91(90.09 \%)$ with mixed type of hearing loss. Chronic Otitis Media Outcome Test-15 (COMOT-15), overall score was between 4150 in $11(10.89 \%)$ patients, 51-60 in $62(61.38 \%)$ patients and Chronic Otitis Media Outcome Test-15 (COMOT-15) overall score was between $61-70$ in $28(27.72 \%)$ patients.

Conclusion: This study showed that speech deficit was severe to profound in majority of patients with Chronic Suppurative Otitis Media. Mixed type of hearing loss is commonest type of deafness and very high scores were observed on COMOT-15 scale, which reflects a significant impairment in quality of life.
\end{abstract}

Keywords: Chronic Suppurative Otitis Media, Pure Tone Audiometry, Speech Recognition Threshold

Article Citation: Nomani T, Rehman AU, Hayat M, Akbar F, Anwawr MA, Shabab MA, Faisal MS. Acoustic and Speech Perceptual Evaluation of health related Quality of Life among adults with Chronic Suppurative Otitis Media. JZMC 2020;11(1):40-45

\section{Introduction}

A chronic inflammation of the middle ear and mastoid cavity is called Chronic Suppurative Otitis Media (CSOM) and it clinically manifests as otorrhoea or recurrent ear discharges via perforation of tympanic membrane. ${ }^{1,2,3}$ The ailment generally instigates in juvenile age by way of a inadvertent perforation of tympanic membrane owing to Acute Otitis Media (AOM) which is a middle ear acute infection. ${ }^{4,5,6}$ In general, when mucoid material ear discharge is continuous from 6 weeks to 12 weeks in an individual with tympanic membrane perforations, even after medical management, this is called CSOM. World Health Organization (WHO) label the individual as patient of CSOM just after 14 days of ear discharge however, otolaryngologists wait for more than three months for the same condition. Clinically, the chronic suppurative otitis media manifests as noteworthy practical restrictions of hearing.7.8.9 In unindustrialized republics, infections of ear and hearing loss are habitually mistreated complaint owing to work force, insufficient funds, knowledge and facilities. 10 However, by advances in hygiene, housing, and use of antibiotic in industrialized republics, the frequency of chronic suppurative otitis media has considerably reduced but it still signifies a hazardous ailment with severe difficulties like cholesteatoma. $^{11,12,13}$ The World Health Organization (WHO) defines the quality of life (QOL) as the individual's perception of his/her position in life, in the context of the culture and value system in which he/she is inserted and in relation to his/her goals, expectations, patterns and worries. ${ }^{14,15}$ The notion of health-related quality of life has evolved since the 1980s as a subjective and multi- dimensional concept that includes domains related to physical, mental,
5. Civil Hospital Bahawalpur, Pakistan.

6. Hitec Institute of Medical Sciences, Texila, Pakistan

7. Sharif Medical City Hospital, Lahore, Pakistan. 
emotional and social functioning. It goes beyond direct measures of population health, life expectancy, causes of death and focuses on the impact of health status on quality of life.

In our country, ear infections and deafness are usually neglected condition due to work force, less facilities, insufficient funds, and lack of knowledge. In rural areas it is more prevalent due to less housing hygiene. If chronic suppurative otitis media persistent it will leads to dangerous disease with severe complications such as cholesteatoma. It is needed to determine the frequency of chronic suppurative otitis media which is more prevalent in population of rural areas. It is also necessary to establish the importance of measuring hearing impairment and speech perception level along with regular medical treatment of disease.

\section{Methodology}

In this cross sectional study, we retrospectively reviewed the data of patients with chronic suppurative otitis media between March, 2015 to September, 2018 performed in ENT/Audiology Departments of District Headquarter Hospital, Hafizabad and Saqib Bashir General Hospital, Hafizabad. The sample size was calculated by Open EPI version 3. We included all the adult patients of age 18-55 years of both gender with diagnosis of Chronic Otitis Suppurative Media was defined as chronic inflammation of the middle ear and mastoid cavity, which presents with recurrent ear discharges or otorrhoea through a tympanic membrane perforation presented to ENT Out Patient Department. We excluded the patients that would be normal or with ear disease other than Chronic Suppurative Otitis Media. We also excluded the individuals with adhesive ear discharge and cholesteatoma. We used the following instruments:

a). COMOT-15: The disease specific health related quality of life was measured with Chronic Otitis Media Outcome Test-15 (COMOT-15), which consists of three sub-scales categorized as Ear symptoms (questions 1-6), Hearing function (questions 7-9), and Physical health (10-13). It was developed by Baumann et al is suitable in this respect as proved by other studies. COMOT-15 questions scores are classified as: no issue at all (0), very mild issue (1), Mild or slight issue (2), Moderate issue (3), Severe issue (4), and Problem as bad as it can be b). Pure Tone Average (PTA): Pure tone air conduction threshold for frequency range of (250$8000 \mathrm{~Hz}$ ) and pure tone bone conduction threshold for frequency range of $(250-4000 \mathrm{~Hz})$ was assessed to find out the degree and type of hearing loss in patients diagnosed with Chronic Suppurative Otitis Media. The results of the Pure tone audiometry was measured as Pure tone average (PTA) which is the average of hearing sensitivity at 500, 1000 and 2000 $\mathrm{Hz}$ frequencies and categorized according to the degree (mild, moderate, moderately severe, severe, profound) and (conductive/mixed) type of hearing loss.

c). Speech Reception threshold (SRT): Speech Reception threshold was measured by speech audiometry at $1000 \mathrm{~Hz}$ frequency on pure tone audiometer. Speech reception threshold is the minimum intensity at which $50 \%$ of the spondee words are repeated correctly by the patient. Spondee words are bisyllabic with equal stress on each syllable. The results of the speech audiometry (speech reception threshold) was categorized according to the degree (mild, moderate, moderately severe, severe and profound) level. Speech reception threshold should be within $10 \mathrm{~dB}$ of the level of pure tone average.

The informed consent, necessary clinical information and demographic profile like age and gender, was taken from all 101 patients. Data was collected in to three steps. First, Pure Tone Audiometry test was performed to access the degree of hearing loss ranged from mild hearing level to moderate, moderately severe, severe and profound hearing level based on Clark classification and the type hearing loss was conductive/mixed. Secondly Speech Audiometry test was performed to access the speech perception level at which the sounds of language are heard, interpreted and understood. The speech perception level was categorized according to the degree (mild, moderate, moderately severe, severe and profound) level. Thirdly, a statement based questionnaire COMOT-15 was used to measure the scoring difference in the all cases. COMOT-15 consists of three categories of questions. First part of questions comprises on disease specific information about ear symptoms and severity. Second part is related to the hearing function. Third part related to physical health in the aspect of communication problems, social withdrawal and impairment of performance. The IBM SPSS 18 was used for data entry and analysis. Qualitative data like gender was presented in the 
form of frequency (percentage) and numerical data like, COMOT-15 test was used to assess the Audiological data (Pure Tone Average and Speech Reception Threshold) scoring differences of individuals was presented in the form of mean standard deviation. Appropriate test was used on the basis of collected data. P-value of less than or equal to 0.05 was considered as significant.

\section{Results}

The mean age of patients with Chronic Suppurative Otitis Media (CSOM) was $33+10$ years. Out of 101 patients with Chronic Suppurative Otitis Media, there were 10 (9.90\%) patients in age group 18-20 years, 37 (36.63\%) patients in age group 21-30 years, $27(26.10 \%)$ patients in age group 31-40 years, $24(23.76 \%)$ patients in age group 41-50 years and there were 3 $(2.97 \%)$ patients in age group 51-55 years. The male to female ratio was $1.1: 1$ in patients with CSOM. Out of 101 patients with Chronic Suppurative Otitis Media, there were $48(47.52 \%)$ females and $53(52.47 \%)$ males. Out of 101 patients with Chronic Suppurative Otitis Media, 9 (8.91\%) patients were students and 34 (33.66\%) patients were house wives. Out of 101 patients with Chronic Suppurative Otitis Media, 19 (18.81\%) patients were doing jobs and 16 (15.84\%) patients were doing business. Out of 101 patients with Chronic Suppurative Otitis Media, $16(15.84 \%)$ patients were farmer and 7 (6.93\%) patients were laborer. The mean duration of disease was $5.6+3.78$ years in patients with Chronic Suppurative Otitis Media. Out of 101 patients with Chronic Suppurative Otitis Media, duration of disease was less than 10 years in 92 $(91.08 \%)$ patients, between 10 to 20 years in 8 (7.92\%) patients and duration of Chronic Suppurative Otitis Media was more than 20 years in $1(0.99 \%)$ patient. The mean Speech Reception Threshold (SRT) score was 83.96-14.93 dBHL in patients with CSOM.

There was no patient with SRT score less than 41 dBHL. Out of 101 patients with Chronic Suppurative Otitis Media, the Speech Reception Threshold (SRT) score was between 41-55 dBHL in $7(6.93 \%)$ patients, between 56-70 dBHL in 17 $(16.83 \%)$ patients, between $71-90 \mathrm{dBHL}$ in 44 $(43.56 \%)$ patients and more than $90 \mathrm{dBHL}$ in 33 $(32.67 \%)$ patients. The distribution of patients by categories of speech deficiency is given in table I.
Table I: Distribution of patients by Speech Deficit Categories

\begin{tabular}{|l|l|l|}
\hline \multirow{2}{*}{ Speech Deficit Categories } & \multicolumn{2}{|l|}{ Number of patients (n=101) } \\
\cline { 2 - 3 } & Frequency & Percentage \\
\hline Mild & 0 & 0.0 \\
\hline Moderate & 7 & 6.93 \\
\hline Moderately severe & 19 & 18.81 \\
\hline Severe & & 41.58 \\
\hline Profound & 42 & 32.67 \\
\hline
\end{tabular}

Table II: Distribution of patients by Categories of Deafness

\begin{tabular}{|l|l|l|}
\hline \multirow{2}{*}{ Deafness Categories } & \multicolumn{2}{|l|}{ Number of patients (n=101) } \\
\cline { 2 - 3 } & Frequency & Percentage \\
\hline Mild & 0 & 0.0 \\
\hline Moderate & 8 & 7.92 \\
\hline Moderately severe & 36 & 35.64 \\
\hline Severe & 44 & 43.56 \\
\hline Profound & 13 & 12.87 \\
\hline
\end{tabular}

Table III: COMOT-15 Overall scores in patients with Chronic Suppurative Otitis Media

\begin{tabular}{|l|l|l|}
\hline \multirow{2}{*}{ COMOrall Score } & \multicolumn{2}{|l|}{ Number of patients (n=101) } \\
\cline { 2 - 3 } & Frequency & Percentage \\
\hline $0-10$ & 0 & 0.0 \\
\hline $11-20$ & 0 & 0.0 \\
\hline $21-30$ & 0 & 0.0 \\
\hline $31-40$ & 0 & 0.0 \\
\hline $41-50$ & 11 & 10.89 \\
\hline $51-60$ & 62 & 61.38 \\
\hline $61-70$ & 28 & 27.72 \\
\hline $71-75$ & 0 & 0.0 \\
\hline Mean + SD & & \multicolumn{2}{|c|}{} \\
\hline
\end{tabular}


The mean Pure Tone Audiometry (PTA) score was $76.48+14.52 \mathrm{~dB}$ in patients with Chronic Suppurative Otitis Media. Out of 101 patients, there was no patient with Pure-Tone Audiometry (PTA) score less than $40 \mathrm{~dB}$. Out of 101 patients with Chronic Suppurative Otitis Media, PureTone Audiometry (PTA) score was between 41-55 $\mathrm{dB}$ in $8(7.92 \%)$ patients, between $56-70 \mathrm{~dB}$ in 30 (29.70\%) patients, between $71-90 \mathrm{~dB}$ in 50 (49.50\%) patients and Pure-Tone Audiometry (PTA) score was more than $90 \mathrm{~dB}$ in 13 (12.87\%) patients. Distribution of patients by categories of deafness is given in table II. Distribution of patients by chronic otitis media outcome test- 15 (COMOT-15) overall score is given in table III.

\section{Discussion}

In this study, the Acoustic and speech perceptual evaluation of health related quality of life among adults with chronic suppurative otitis media were assessed. The mean age of patients with Chronic Suppurative Otitis Media was $33+10$ years in our study. The majority of patients with Chronic Suppurative Otitis Media in our study was in age group 21-30 years i.e. $36.63 \%$ followed by age group $31-40$ years $(26.10 \%)$, age group $41-50$ years $(23.76 \%)$, age group $18-20$ years $(9.90 \%)$ age group $51-55$ years $(2.97 \%)$. In study by Alabbasi AM et al. ${ }^{16}$ It was found that CSOM was most common among infants and children i.e. $60 \%$. In a study by Kolo ES et al. ${ }^{17}$ The mean age of patients was $28.93 € 18.593$ years (Range: 4-73 years). In a study by Baumann I et al 18 the median age of patients was 48 years (range 18-75 years). In our study, the male predominance was observed i.e. $52.47 \%$ males and $47.52 \%$ females with male to female ratio of $1.1: 1$ in patients with Chronic Suppurative Otitis Media. Male dominance was also observed in study by Alabbasi AM et al16 there were $65(54.16 \%)$ males and $55(45.83 \%)$ females, and males to females ratio was 1.2:1. However, female dominance was observed in a study by Baumann I et al 18 i.e. 58 males and 63 females (Baumann I et al., 2011). In another study by Lasisi $\mathrm{AO}$ et al, 19 there were 173 girls and 116 boys with M:F 1.5:1. In a cross-sectional prospective study Maharjan $\mathrm{M}$ et al, ${ }^{20}$ there were 44 males and 56 females. In our study, there were $8.91 \%$ students, $33.66 \%$ house wives, $15.84 \%$ farmer, $6.93 \%$ laborer and $18.81 \%$ patients were doing jobs while $15.84 \%$ patients were doing business. In a prospective study of hearing loss in CSOM by Lasisi AO et al, of the 89 patients, 61 $(69 \%)$ patients were belonged to low social class, $13 / 37(35 \%)$ were middle class and 15/63 (24\%) upper class. 19 In a cross-sectional study by Olatoke $\mathrm{F}$ et al, $21 \mathrm{CSOM}$ was present in 35 children $(2.3 \%)$, 12 from the low-status school (4.0\%), 11 from the middle-status school (2.8\%), and 12 from the highstatus school (1.5\%); the overall difference in prevalence among the three schools was statistically significant $(x=6.40$; degrees of freedom $[\mathrm{df}]=2 ; \mathrm{p}=$ $0.04)$. In our study, the mean duration of disease was $5.6+3.78$ years in patients with chronic suppurative otitis media. The duration of disease was less than 10 years in majority of patients in our study i.e. $91.08 \%$ patients. In a study by Kolo ES et al, 17 the duration of otorrhoea ranged from 2 months to 20 years; with a mean of $6.11=6.393$ years. In a prospective study of hearing loss in CSOM by Lasisi AO et al, duration of CSOM ranged from 4 weeks to 12 years $(4+2.04$ years) and from 5 to 12 years in those with SHL (94 6.21 years). In a cross- sectional prospective study Maharjan $\mathrm{M}$ et al, 20 duration of ear discharge was less than 10 years in $38 \%$ patients and duration was more than 10 years in $62 \%$ patients.

The mean Speech Reception Threshold (SRT) score in our study was 83.96-14.93 dBHL in patients with chronic suppurative otitis media. In our study, there was no $0.0 \%$ ) patient with SRT score less than 41 dBHL. The majority of patients in our study had Speech Reception Threshold (SRT) score between 71-90 dBHL i.e. $43.56 \%$ patients. In our study, the speech deficit was moderate in $7(6.93 \%)$ patients, moderately severe in $19(18.81 \%)$, severe in 42 $(41.58 \%)$ and speech deficit was profound in 33 $(32.67 \%)$ patients. There was no patient $(0.0 \%)$ with mild speech deficit in our study. In our study, the mean Pure Tone Audiometry (PTA) score was $76.48+14.52 \mathrm{~dB}$ in patients with chronic suppurative otitis media. The majority of patients had Pure Tone Audiometry (PTA) score between 71-90 dB i.e. $49.50 \%$ patients in our study. There was no patient $(0.0 \%)$ with Pure-Tone Audiometry (PTA) score less than $40 \mathrm{~dB}$ in our study. In a study by Kolo ES et al, 17 the mean bone conduction threshold in the diseased ear was $39.07-12.028 \mathrm{~dB}$ and $10.26+2.620 \mathrm{~dB}$ in the control ear $(\mathrm{P}<0.05)$. The mean bone conduction threshold differences between the diseased and control ears range from 21.69 to $34.52 \mathrm{~dB}$ across the frequencies $0.5,1.0,2.0$, and $4.0 \mathrm{kHz}$. These differences tended to increase with increasing frequency and were all significant $(\mathrm{P}<0.05)$. 
(Kolo ES et al., 2012). In a cross-sectional prospective study Maharjan M et al, 20 analysis of the pure tone audiogram of 96 patients (80.67\%) showed more loss in low frequencies with larger air bone gap at low frequencies. Remaining 23 patients (19.32\%) showed loss involving all the frequencies. In our study, the deafness was moderate in $8(7.92 \%)$ patients, moderately severe in $36(35.64 \%)$ patients, severe in 44 (43. 56\%) patients and deafness was profound in 13 $(12.87 \%)$ patients with chronic suppurative otitis media. There was no patient $(0.0 \%)$ with mild deafness in our study. In a cross-sectional study by Olatoke F et al, ${ }^{21} 18$ (34.6\%) had a pure-tone average (PTA) within normal limits, 20 (38.5\%) had a mild conductive hearing loss, and 14 $(26.9 \%)$ had a moderate loss. In a cross-sectional prospective study Maharjan $\mathrm{M}$ et $\mathrm{al},{ }^{20}$ hearing status of all 119 perforated tympanic membrane is mild loss 29 (24.37\%), moderate loss 63 (52.94\%), moderate severe loss $24(20.17 \%)$ and severe loss $3(2.52 \%)$.

In our study, there were $10(9.90 \%)$ patients with conductive type of hearing loss and 91 (90.09\%) patients with mixed type of hearing loss. There was no patient $(0.0 \%)$ patients with sensorineural type of hearing loss in our study. In a study by Taipale A et al,22 hearing impairment of $>25 \mathrm{~dB}$ was present in $52 \%$ of CSOM affected ears and bilateral hearing loss in $7(30 \%)$ CSOM children vs. zero control child $(\mathrm{p}=0.009)$. In study by Alabbasi AM et al, ${ }^{16}$ it was found that the most common type was conductive hearing loss $(55.5 \%)$ followed by mixed hearing loss $(27.7 \%)$ and sensorineural hearing loss $(16.6 \%)$. However, in a study by Kolo ES et al, ${ }^{17}$ the patients with chronic suppurative otitis media had a significant degree of sensorineural hearing loss. In a prospective study of hearing loss in CSOM by Lasisi $\mathrm{AO}$ et al, the prevalence of hearing loss in CSOM was 89/189(47\%) and was conductive in $73 / 89$ (82\%) and sensorineural (SHL) in 16/89 $(18 \%){ }^{16}$ In our study, Chronic Otitis Media Outcome Test-15 (COMOT-15) Overall score was between $41-50$ in $11(10.89 \%)$ patients, 51-60 in $62(61.38 \%)$ patients and Chronic Otitis Media Outcome Test-15 (COMOT-15) Overall score was between $61-70$ in $28(27.72 \%)$ patients.Chronic Otitis Media Outcome Test-15 (COMOT-15) Overall score was not less than 41 and not more than 70 in any $(0,0 \%)$ patient in our study. In a study by Baumann I et al, ${ }^{23}$ baseline Chronic Otitis Media Outcome Test-15 (COMOT-15) Overall score was $46.6+18.8$ and COMOT-15 Overall score was $38.4+20.5$ at 6 months and $39.5+22.0$ at 12 months. Our study reflects that COMOT score has been highest in majority of cases. Out of total $(89.10 \%)$ patients had COMOT score higher than 50.80 that reflects the need of conducting COMOT test immediately along with the clinical evaluation of patients. This can simply be performed on regular basis in OPD by completing a simple Performa's as is done by researcher. We have assessed the rural population of a DHQ Hospital where management of patients did not present to the hospital or doctor unless there is some emergency. The commonest complaints of chronic otitis media was ear discharge which is locally managed by quacks or Hakeem's or by folk medication. So the true number of cases may be higher. In this regard we need to run more studies and ENT specialist must be aware of importance of hearing and speech assessment and should work in close collaboration with Audiologist. In time referral may help in reducing the morbidity associated with CSOM. This is the first national study to assess the Acoustic and speech perceptual evaluation of health related quality of life among adults with Chronic Suppurative Otitis Media. This study is limited by a small number of patients. Further studies should be conducted in the future. We applied COMOT for evaluation of quality of life. The interpretations are relied upon maximum score or different score groups. However there is need to improve the scoring system of COMOT-15 by grading the sum of scores.

\section{Conclusion}

The results of this study concluded that speech deficiency was severe to profound in majority of patients with chronic suppurative otitis media. Mixed type of hearing loss is commonest type of deafness in Chronic Suppurative Otitis Media. Very high scores were observed on COMOT-15 scales which reflects a significant impairment in quality of life among patients with Chronic Suppurative Otitis Media.

Authors Contribution: TN: Design of work, Acquisition and analysis of data and drafting. AR: Interpretation of data, revising. MH \& FA: Design of work, Acquisition and analysis of data. . MAA: Interpretation of data, revising. SAS \& MSF: Conception of work, design of work and revising.

Conflict of interest: None

Resources of funding: Self 


\section{References}

1. Neilan RE, Isaacson B, Kutz JW Jr, Lee KH, Roland PS. Pediatric otogenic lateral sinus thrombosis recanalization. Int $\mathrm{J}$ Pediatr Otorhinolaryngol 2011;75(6):850-3.

2. Osma U, Cureoglu S, Hosoglu S. The complications of chronic otitis media: report of 93 cases. J Laryngol Otol 2000; 114(2): 97-100.

3. Mittal R, Parrish JM, Soni M, Mittal J, Mathee K. Microbial otitis media: recent advancements in treatment, current challenges and opportunities. J Med Microbiol 2018; 67(10): 1417-25.

4. Magsi PB, Jamro B, Sangi HA. Clinical presentation and outcome of mastoidectomy in chronic suppurative otitis media (CSOM) at a tertiary care hospital Sukkur, Pakistan. Rawal Med J 2012; 37(1): 50-3.

5. Park J, Cheng JT, Ferguson D, Maguluri G, Chang EW, Clancy C, Lee DJ, Iftimia N. Investigation of middle ear anatomy and function with combined video otoscopyphase sensitive OCT. Biomed Opt Express 2016; 7(2): 238-50.

6. Prabhu P, Ganeshan A, Jose A, Kiruthika L. Healthrelated quality of life measurement in individuals with chronic suppurative otitis media: a South Indian perspective. Hear Balance Commun 2017; 15(3): 15660.

7. Samal DK, kumarSahoo A, Sarkar S. Obstructive Hydrocephalous: An Unusual Complication of Chronic Suppurative Otitis Media. Otolaryngol Head Neck Surg 2017; 156: 88-105.

8. Happani H, Kalola J, Rathod H, Trivedi A. Role of HRCT Temporal Bone In Patients with Chronic Supparative Otitis Media. Radiol 2018; 3(3): 70-2.

9. Jensen RG, Koch A, Homoe P. The risk of hearing loss in a population with a high prevalence of chronic suppurative otitis media. Int J Pediatr Otorhinolaryngol 2013; 77(9): 1530-5.

10. Singer AEA, Abdel-NabyAwad OG, El-Kader RMA, Mohamed AR. Risk factors of sensorineural hearing loss in patients with unilateral safe chronic suppurative otitis media. Am J Otolaryngol 2018; 39 (2): 88-93.

11. Wang B, Cheng Y, Xu M. Characterization of the T-cell subpopulations in the granulation tissues of chronic suppurative otitis media. Biomed Rep. 2016;4(6):694-8.

12. Wang PC, Nadol JB, Merchant S, et al. Validation of outcomes survey for adults with chronic suppurative otitis media. Ann Otol Rhinol Laryngol 2009; 109: 24954.
13. Luers JC, Hüttenbrink KB. Surgical anatomy and pathology of the middle ear. J Anat 2016; 228(2): 338-53.

14. Pichora-Fuller MK, Singh G. Effects of age on auditory and cognitive processing: implications for hearing aid fitting and audiologic rehabilitation. Trends Amplif 2006; 10(1): 29-59.

15. Justin R, Tumweheire G, Kajumbula H, Ndoleriire C. Chronic suppurative otitis media: bacteriology, susceptibility and clinical presentation among ENT patients at Mulago Hospital, Uganda. South Sudan Med J 2018; 11(2): 31-5.

16. Alabbasi AM, Alsaimary IE, Najim JM. Prevalence and patterns of chronic suppurative otitis media and hearing impairment in Basrah city. J Med Med Sci 2010; 1(4): 12933.

17. Kolo ES, Salisu AD, Yaro AM, Nwaorgu OG. Sensorineural hearing loss in patients with chronic suppurative otitis media. Indian J Otolaryngol Head Neck Surg 2012; 64(1): 59-62.

18. Baumann I, Gerendas B, Plinkert PK, Praetorius M. General and disease-specific qualityof life in patients with chronic suppurative otitis media-a prospective study. Health Qual Life Out 2011; 9(1): 48-53

19. Lasisi AO, Sulaiman OA, Afolabi OA. Socio-economic status and hearing loss inchronic suppurative otitis media in Nigeria. Ann Trop Paediatr 2007; 27(4): 291-6.

20. Maharjan M, Kafle P, Bista M, Shrestha S, Toran KC. Observation of hearing loss in patients with chronic suppurative otitis media tubotympanic type. Kathmandu Uni Med J 2009; 7(4): 397-401.

21. Olatoke F, Ologe FE, Nwawolo CC, et al; The prevalence of hearing loss among schoolchildren with chronic suppurative otitis media in Nigeria, and its effect on academic performance. Ear Nose Throat J 2008; 87(12): 19.

22. Taipale A, Pelkonen T, Taipale M, Bernardino L, Peltola H, Pitkäranta A. Chronic suppurative otitis media in children of Luanda, Angola. Acta Paediatrica 2011; 100(8): 84-8.

23. Baumann I, Gerendas B, Plinkert PK, Praetorius M. General and disease-specific quality of life in patients with chronic suppurative otitis media-a prospective study. Health Qual Life Out 2011; 9(1):48-53 